\title{
THE HISTORY OF NEUTRINO OSCILLATIONS ${ }^{1}$
}

\author{
S. M. Bilenky \\ Joint Institute for Nuclear Research, Dubna, R-141980, Russia \\ SISSA, via Beirut 2-4, I-31014, Trieste, Italy
}

\begin{abstract}
The early history of neutrino masses, mixing and oscillations is briefly reviewed.
\end{abstract}

\section{Introduction}

After many years of heroic efforts of many physicists we have now model independent evidence of neutrino oscillations. The evidence of neutrino oscillations was obtained in the atmospheric Super Kamiokande experiment [1, in the solar SNO experiment [2] in the reactor KamLAND experiment [3, and also in solar neutrino experiments [4, 5, 6, 7], atmospheric neutrino experiments [8, 9], and in the first long baseline accelerator K2K experiment 10].

Neutrino oscillations are signature of small neutrino masses and neutrino mixing. It took more than 40 years to discover this phenomenon.

The first idea of neutrino oscillations was put forward by B. Pontecorvo in 1957-58 [11, 12. I worked with B. Pontecorvo more than 15 years, starting from the time when majority of physicists believed that neutrinos are massless two-component particles. I will consider mainly the evolution of original ideas of neutrino masses, mixing and oscillations.

When Pauli introduced neutrino in 1930 he assumed that neutrino ("neutron") is a neutral weakly interacting particle with spin $1 / 2$ and a mass smaller than electron mass. The first method of the measurement of neutrino mass was proposed in 1933 by Fermi [13] and Perrin [14. They proposed to search for effects of neutrino mass via detailed investigation of the highenergy part of $\beta$-spectra which correspond to the emition of neutrino with a small energy.

\footnotetext{
${ }^{1}$ A report at the "Nobel Symposium on Neutrino physics", Haga Slott, Enkoping, Sweden, August 19-24, 2004
} 
Usually effect of the neutrino mass is searched for through the investigation of the $\beta$-spectrum of the decay

$$
{ }^{3} \mathrm{H} \rightarrow{ }^{3} \mathrm{He}+\mathrm{e}^{-}+\bar{\nu}_{\mathrm{e}}
$$

Up to now no effects of neutrino mass was found in these experiments. In the first experiments for the upper bound of the neutrino mass it was obtained [15]

$$
m_{\nu} \lesssim 500 \mathrm{eV}
$$

With further experiments this bound was decreasing and at the end of the fifties for the upper bound of the neutrino mass was found the value

$$
m_{\nu} \lesssim(100-200) \mathrm{eV} .
$$

The two-component neutrino theory, proposed by Landau [16], Lee and Yang [17 and Salam [18] in 1957 after the violation of the parity in the $\beta$-decay was discovered [19], was first theoretical idea about neutrino mass.

In order to demonstrate the idea of the two-component neutrino let us consider the Dirac equation for the field of neutrino with mass $m_{\nu}$

$$
i \gamma^{\alpha} \partial_{\alpha} \nu(x)-m_{\nu} \nu(x)=0
$$

For left-handed and right-handed components $\nu_{L}(x)$ and $\nu_{R}(x)$ from Eq.(3) we have two coupled equations

$$
i \gamma^{\alpha} \partial_{\alpha} \nu_{L}(x)-m_{\nu} \nu_{R}(x)=0
$$

and

$$
i \gamma^{\alpha} \partial_{\alpha} \nu_{R}(x)-m_{\nu} \nu_{L}(x)=0
$$

Taking into account the bound (2) it looked natural at the fifties that neutrino mass is equal to zero. This assumption was made by Landau, Lee and Yang and Salam.

For $m_{\nu}=0$ from (44) and (51) we obtain two decoupled Weil equations

$$
i \gamma^{\alpha} \partial_{\alpha} \nu_{L, R}(x)=0
$$

and the neutrino field can be in this case

$$
\nu_{L}(x) \text { or } \nu_{\mathrm{R}}(\mathrm{x}) .
$$

The was the choice of the authors of the two-component neutrino theory.

If neutrino field is $\nu_{L}(x)\left(\nu_{R}(x)\right)$ 
1. The general Hamiltonian of the $\beta$-decay has the form

$$
\mathcal{H}_{I}^{\beta}=\sum_{i} G_{i}\left(\bar{p} O_{i} n\right)\left(\bar{e} O^{i} \frac{1}{2}\left(1 \mp \gamma_{5}\right) \nu\right)+\text { h.c. },
$$

where the index $i$ runs over $S, V, T, A, P$ (scalar, vector etc)

Thus, the two-component neutrino theory ensure large violation of parity, observed in the $\beta$-decay.

2. Neutrino helicity is equal to $-1(+1)$ and antineutrino helicity is equal to $+1(-1)$ in the case of $\nu_{L}(x)\left(\nu_{R}(x)\right)$.

Neutrino helicity was measured in 1958 in a spectacular M.Goldhaber et al experiment [20]. In this experiment the circular polarization of $\gamma$-quanta from the chain of the reactions

$$
\begin{aligned}
e^{-}+\mathrm{Gd} \rightarrow \nu_{\mathrm{e}}+ & \mathrm{Sm}^{*} \\
& \downarrow \\
& \mathrm{Sm}+\gamma
\end{aligned}
$$

was measured. The measurement of the polarization of the $\gamma$-quanta allowed to determine the longitudinal polarization of neutrino. It was found that neutrino is the left-handed particle. Thus, the neutrino field is $\nu_{L}(x)$.

It is interesting to notice that equations (6) for a massless particle was discussed by Pauli in his encyclopedia article "General Principles of Quantum Mechanics" (1933). Pauli wrote that because equation for $\nu_{L}\left(\nu_{R}\right)$ is not invariant under space reflection it is "not applicable to the physical reality".

From the point of view of the two-component theory large violation of parity in the $\beta$-decay and other leptonic processes is ultimately connected with equal to zero neutrino mass. This point of view changed after Feynman and Gell-Mann [21], Marshak and Sudarshan [22] proposed in $1958 \mathrm{~V}-\mathrm{A}$ theory.

This theory was based on the assumption that in the Hamiltonian of the weak interaction enter left-handed components of all fields. This means that the violation of parity in the weak interaction is not connected with exceptional properties of neutrinos. Exist other reasons for left-handed fields in the Hamiltonian. Moreover, after the V-A theory it was natural to turn up arguments and consider neutrino as a particle with different from zero mass (see later). 
Nevertheless, the two-component neutrino theory was a nice and the simplest theoretical possibility. It was in a perfect agreement with numerous experiments on the investigation of weak processes. From my point of view this was the main reason why during many years there was a common opinion that neutrinos are massless particles. The Glashow-Weinberg-Salam Standard Model was build under the assumption of massless two-component neutrinos.

\section{B. Pontecorvo}

The first idea of neutrino masses, mixing and oscillations was suggested by B.Pontecorvo in 1957 [11. He thought that there is an analogy between leptons and hadrons and he believed that in the lepton world exist phenomenon analogous to the famous $K^{0} \rightleftarrows \bar{K}^{0}$ oscillations.

The only possible candidate were neutrino oscillations. At that time only one neutrino type was known. Possible oscillations in this case are

$$
\nu_{L} \rightleftarrows \bar{\nu}_{L} \text { and } \quad \bar{\nu}_{\mathrm{R}} \rightleftarrows \nu_{\mathrm{R}}
$$

According to the two-component neutrino theory the states $\bar{\nu}_{L}$ and $\nu_{R}$ do not exist. Such states were a problem for B. Pontecorvo. We will see how he solved it.

In 1957-58 R.Davis 23] was doing an experiment searching for production of ${ }^{37} \mathrm{Ar}$ in the process

$$
\bar{\nu}_{e}+{ }^{37} \mathrm{Cl} \rightarrow \mathrm{e}^{-}+{ }^{37} \mathrm{Ar}
$$

with antineutrinos from a reactor. A rumor reached B.Pontecorvo that Davis observed production of ${ }^{37}$ Ar. B. Pontecorvo, who was thinking about neutrino oscillations at that time, decided that production of ${ }^{37} \mathrm{Ar}$ could be due to antineutrino $\rightleftarrows$ neutrino transitions in vacuum. He published the paper on neutrino oscillations [12. In this paper he wrote:

"Recently the question was discussed whether there exist other mixed neutral particles beside the $K^{0}$ mesons, i.e. particles that differ from the corresponding antiparticles, with the transitions between particle and antiparticle states not being strictly forbidden. It was noted that neutrino might be such a mixed particle, and consequently, there exists the possibility of real neutrino $\rightleftarrows$ 
antineutrino transitions in vacuum, provided that lepton (neutrino) charge is not conserved. This means that the neutrino and antineutrino are mixed particles, i.e., a symmetric and antisymmetric combination of two truly neutral Majorana particles $\nu_{1}$ and $\nu_{2} . "$

\section{B.Pontecorvo came to the conclusion that}

"The flux of neutral leptons consisting mainly of antineutrino when emitted from a reactor will consist at some distance $\mathrm{R}$ from the reactor of half neutrinos and half antineutrinos"

In the fifties Reines and Cowan 24 were doing their famous experiment in which $\bar{\nu}_{e}$ was discovered via the observation of $e^{+}$and neutrons produced in the reaction

$$
\bar{\nu}_{e}+p \rightarrow e^{+}+n
$$

In order to see effect of neutrino oscillations B. Pontecorvo proposed that

"It will be extremely interesting to perform Cowan and Reines experiment at different distances from reactor"

In the paper [12] which was written at the time when the two-component theory had just appeared and the Davis experiment was not finished B. Pontecorvo wrote

"...it is not possible to state apriori that some part of the flux can initiate the Davis reaction"

Thus, he admitted at that time that two-component theory can be violated. Later after the Davis experiment was finished and no production of ${ }^{3} 7 \mathrm{Ar}$ was observed B. Pontecorvo understood that due to oscillations neutrino (antineutrino) could transfer into $\bar{\nu}_{L}\left(\nu_{R}\right)$, particles which do not participate in the standard weak interaction. B. Pontecorvo was the first who introduced the notion of sterile neutrinos so popular nowadays.

After the second neutrino $\nu_{\mu}$ was discovered in the Brookhaven experiment 25] it was very natural (and not difficult ) for B. Pontecorvo to generalize his idea of neutrino oscillations for the case of two neutrinos [26]. He considered in the paper [26] oscillations into active and sterile states $\nu_{\mu} \rightleftarrows \nu_{e}$, $\nu_{\mu} \rightleftarrows \bar{\nu}_{\mu L}$ etc. 
In the paper [26] B. Pontecorvo discussed oscillations of the solar neutrinos. In 1967 the Davis solar neutrino experiment only started. Three years before the first results of Davis experiment were published B. Pontecorvo pointed out that due to neutrino oscillations the observed flux of solar neutrinos could be two times smaller than the expected flux.

"From an observational point of view the ideal object is the sun. If the oscillation length is smaller than the radius of the sun region effectively producing neutrinos, (let us say one tenth of the sun radius $R_{\odot}$ or 0.1 million $\mathrm{km}$ for ${ }^{8} B$ neutrinos, which will give the main contribution in the experiments being planned now), direct oscillations will be smeared out and unobservable. The only effect on the earth's surface would be that the flux of observable sun neutrinos must be two times smaller than the total neutrino flux".

In the Davis experiment only very rare high energy solar neutrinos mainly from the decay ${ }^{8} B \rightarrow{ }^{8} B e^{+} \nu_{e}$ can be observed. In [26] B. Pontecorvo wrote

"Unfortunately, the relative weight of different thermonuclear reactions in the sun and its central temperature are not known well enough to permit a comparison of the expected and observed solar neutrino intensities"

In 1967 it was impossible to envisage the NC result of the SNO experiment 2 in which the total flux of the ${ }^{8} B$ neutrinos was measured. At that time even neutral current interaction was not known. It is interesting to notice that in 1988 when the SNO experiment was in its initial stage B.Pontecorvo enthusiastically supported the experiment. This is a letter which B. Pontecorvo wrote at that time.

Dr. Walter F. Davidson

High Energy Physics Section, National Research Council, Canada

Dear Dr. Davidson,

Thank you very much for sending me the SNO proposal. Below I am writing a short comment on SNO in the hope that opinion of a person who already in 1946 worked in Canada on neutrinos may be of some value. The SNO proposal (1000 tons of $\mathrm{D}_{2} \mathrm{O}$ immersed 
in $\mathrm{H}_{2} \mathrm{O}$ in a mine $2 \mathrm{~km}$ deep) in my opinion is a wonderful proposal for several reasons.

First it is new in the sense that with the help of large $\mathrm{D}_{2} \mathrm{O}$ detector immersed in $\mathrm{H}_{2} \mathrm{O}$ there becomes possible the investigation of reactions 1. $\nu_{e} d \rightarrow e^{-} p p 2$. $\nu_{x} e \rightarrow \nu_{x} e 3 . \nu_{x} d \rightarrow \nu_{x} n p 4 . \bar{\nu}_{e} d \rightarrow e^{+} n n$ 5. $\bar{\nu}_{e} p \rightarrow e^{+} n$ with main application to solar and star collapse neutrinos $(1,2,3)$ and star collapse antineutrinos $(4,5)$.

Second. the proposal is realistic, in a sense that at least one large Cerenkov counter filled with $\mathrm{H}_{2} \mathrm{O}$ is known to work properly (Kamiokande)

Third, the proposal can be realized only in Canada, where for historical reasons large quantities of $\mathrm{D}_{2} \mathrm{O}$ are available during a period of several years.

Finally, in my opinion the neutral current reaction 3. yielding the total number of neutrinos of all flavors, can be investigated in spite of serious difficulties of registration of neutrons.

In conclusion the SNO proposal is progressive and should be supported by all means.

Yours sincerely,

Bruno Pontecorvo,

Dubna August 18, 1988.

\section{Z.Maki, M. Nakagawa and S. Sakata}

Two-neutrino mixing was proposed in 1962 by Z. Maki,M.Nakagawa and S. Sakata in a paper [27] which was practically unknown for many years. The approach accepted in this paper was based on the Nagoya model. According to this model in hadronic current enter fields of three fundamental barions $p, n$ and $\Lambda$. These particles were considered as bound states of leptons and a boson $B^{+}$("new sort of matter"):

$$
p=<\nu B^{+}>, n=<e^{-} B^{+}>, \Lambda=<\mu^{-} B^{+}>.
$$

A natural consequence of the model was barion-lepton symmetry, a symmetry of the weak current under the exchange

$$
\nu \leftrightarrow p, \quad e^{-} \leftrightarrow n, \quad \mu^{-} \leftrightarrow \Lambda
$$


The paper [27] was writen at the time when there was an indication 28], obtained from the analysis of the data of experiments on the search for $\mu \rightarrow e \gamma$, that $\nu_{e}$ and $\nu_{\mu}$ are different particles. The Brookhaven neutrino experiment [25] which proved that $\nu_{e}$ and $\nu_{\mu}$ are different particles was in preparation at that time.

Possible existence of two different neutrinos was a problem for the Nagoya model (four leptons and three fundamental hadrons). MNS proposed the following solution of the problem. The leptonic weak current

$$
j_{\alpha}=2\left(\bar{\nu}_{e L} \gamma_{\alpha} e_{L}+\bar{\nu}_{\mu L} \gamma_{\alpha} \mu_{L}\right)
$$

determines weak neutrinos $\nu_{e}$ and $\nu_{\mu}$. They wrote

"The definition of the particle state of neutrino is quite arbitrary; we can speak of "neutrinos" which are different of weak neutrinos but expressed by the linear combinations of the latter. We assume that there exist a representation which defines the true neutrinos $\nu_{1}$ and $\nu_{2}$ through orthogonal transformation:

$$
\begin{aligned}
& \nu_{1}=+\nu_{e} \cos \delta+\nu_{\mu} \sin \delta \\
& \nu_{2}=-\nu_{e} \sin \delta+\nu_{\mu} \cos \delta
\end{aligned}
$$

The true neutrinos should be so defined that $B^{+}$can be bound to $\nu_{1}$ but can not be bound to $\nu_{2}$ "

Thus, MNS proposed modified Nagoya model:

$$
p=<\nu_{1} B^{+}>, n=<e^{-} B^{+}>, \Lambda=<\mu^{-} B^{+}>
$$

To the lepton current, written in terms of "true neutrinos",

$$
j_{\alpha}=2\left(\bar{\nu}_{1 L} \gamma_{\alpha} e_{L} \cos \delta+\bar{\nu}_{1 L} \gamma_{\alpha} \mu_{L} \sin \delta\right)+\ldots
$$

corresponds the hadronic current

$$
j_{\alpha}=2\left(\bar{p}_{L} \gamma_{\alpha} n_{L} \cos \delta+\bar{p}_{L} \gamma_{\alpha} \Lambda_{L} \sin \delta\right)+\ldots
$$

which was identical to Gell-Mann-Levy current [29]. In fact this was a preCabibbo discussion of the hadron mixing. 
Further, MNS assumed that there exist an additional interaction of $\nu_{2}$ with a field $X$ of heavy bosons

$$
\mathcal{L}=g \bar{\nu}_{2} \nu_{2} X^{+} X
$$

which provide difference of the masses of $\nu_{2}$ and $\nu_{1}$.

In connection with the Brookhaven neutrino experiment MNS wrote

"Weak neutrinos ${ }^{2}$

$$
\begin{aligned}
& \nu_{e}=\nu_{1} \cos \delta-\nu_{2} \sin \delta \\
& \nu_{\mu}=\nu_{1} \sin \delta+\nu_{2} \cos \delta
\end{aligned}
$$

are not stable due to occurrence of virtual transition $\nu_{e} \leftrightarrow \nu_{\mu}$ caused by the interaction (14). A chain of reactions

$$
\begin{array}{r}
\pi^{+} \rightarrow \mu^{+}+\nu_{\mu} \\
\nu_{\mu}+Z \rightarrow Z^{\prime}+\left(\mu^{-} \text {and } / \text { or } \mathrm{e}^{-}\right)
\end{array}
$$

is useful to check the two-neutrino hypothesis only when $\mid m_{\nu_{2}}-$ $m_{\nu_{1}} \mid \leq 10^{-6} \mathrm{MeV}$ under the conventional geometry of the experiments . Conversely, the absence of $e^{-}$will be able not only to verify two-neutrino hypothesis but also to provide an upper limit of the mass of the second neutrino $\nu_{2}$ if the present scheme should be accepted"

\section{B. Pontecorvo and collaborators}

The first phenomenological theory of two-neutrino mixing was proposed by V. Gribov and B. Pontecorvo in 1969 [30]. They assumed that the lefthanded fields $\nu_{e L}$ and $\nu_{\mu L}$ enter not only into the weak interaction but also into a mass term (which they called an additional neutrino interaction).

Let us notice that there was a wide-spread prejudice at that time that in the case of left-handed neutrino fields neutrino masses must be equal to zero. This is correct if total lepton number is conserved. In [30] it was shown that if total lepton number is changed by 2 neutrino masses can be introduced even in the case of left-handed fields in the Lagrangian.

\footnotetext{
${ }^{2}$ Let us stress that according to MNS $\delta$ is the Cabibbo angle.
} 
Gribov and Ponecorvo assumed that the following neutrino mass term enters into the Lagrangian

$$
\begin{aligned}
& \mathcal{L}^{\mathrm{M}}=-\frac{1}{2}\left(m_{e \bar{e}} \overline{\left(\nu_{e L}\right)^{c}} \nu_{e L}+m_{\mu \bar{\mu}} \overline{\left(\nu_{\mu L}\right)^{c}} \nu_{\mu L}+\right. \\
& \left.\left.m_{e \bar{\mu}}\left(\overline{\left(\nu_{e L}\right)^{c}} \nu_{\mu L}+\overline{(} \nu_{\mu L}\right)^{c} \nu_{e L}\right)\right)+ \text { h.c. }
\end{aligned}
$$

Here $m_{\mu \bar{\mu}}, \quad m_{e \bar{e}}$ and $m_{e \bar{\mu}}$ are real parameters and $\left(\nu_{l L}\right)^{c}=C\left(\bar{\nu}_{l L}\right)^{T}$ is the conjugated field.

After the diagonalization of the mass term (16) the following mixing relations were obtained

$$
\begin{gathered}
\nu_{e L}=\cos \theta \nu_{1 L}+\sin \theta \quad \nu_{2 L} \\
\nu_{\mu L}=-\sin \theta \nu_{1 L}+\cos \theta \nu_{2 L} .
\end{gathered}
$$

Here $\nu_{1,2}$ are fields of Majorana neutrinos with masses $m_{1,2}$. The mass term (16) is called the Majorana mass term.

Possible oscillations in the case of the mixing (17) are $\nu_{e} \rightleftarrows \nu_{\mu}$ and $\bar{\nu}_{e} \rightleftarrows$ $\bar{\nu}_{\mu}$. There are no transitions into sterile states in the case of the Majorana mass term. This was one of the main idea of Gribov and Pontecorvo.

It was shown in 30] that the observables (the mixing angle $\theta$ and Majorana neutrino masses $m_{1,2}$ ) are connected with the parameters of the theory by the relations

$$
\begin{gathered}
\tan 2 \theta=\frac{2 m_{e \bar{\mu}}}{m_{\mu \bar{\mu}}-m_{e \bar{e}}}, \\
m_{1,2}=\frac{1}{2}\left|m_{\mu \bar{\mu}}+m_{e \bar{e}} \mp \sqrt{\left(m_{\mu \bar{\mu}}-m_{e \bar{e}}\right)^{2}+4 m_{e \bar{\mu}}^{2}}\right| .
\end{gathered}
$$

From (18) it follows that if $\mu-e$ symmetry

$$
m_{\mu \bar{\mu}}=m_{e \bar{e}} ; \quad m_{e \bar{\mu}} \neq 0
$$

holds, $\theta=\pi / 4$ (maximal mixing). Vacuum oscillations of solar neutrinos were discussed in 30] in this case.

The full phenomenological theory of neutrino mixing and the theory of neutrino oscillations in vacuum were developed in the seventies.

In the papers [31] neutrino mixing was introduced in analogy with CabibboGIM mixing of quarks (lepton-quark analogy).

The main idea of the paper [31] was the following: neutrinos like all other fundamental fermions (leptons and quarks) are massive particles. A mixing 
of massive fermions is a general feature of gauge theories with spontaneous violation of symmetry. Thus, it is natural to assume that phenomenon of mixing is common for quarks and massive neutrinos. For the case of two neutrinos we have

$$
\begin{aligned}
& \nu_{e L}=\cos \theta \nu_{1 L}+\sin \theta \nu_{2 L} \\
& \nu_{\mu L}=-\sin \theta \nu_{1 L}+\cos \theta \nu_{2 L}
\end{aligned}
$$

Here $\nu_{1,2}$ are 4-component fields of Dirac neutrinos with masses $m_{1,2}$.

Possible values of the neutrino mixing angle were discussed in [31. We have concluded

"... it seems to us that the special values of the mixing angle $\theta=0$

(the usual scheme in which muonic charge is strictly conserved) and $\theta=\pi / 4$ (maximal mixing) are of the greatest interest."

The Dirac mass term has the form

$$
\mathcal{L}^{\mathrm{D}}=-\sum_{l l^{\prime}} \bar{\nu}_{l^{\prime} R} M_{l^{\prime} l}^{\mathrm{D}} \nu_{l L}+\text { h.c. }
$$

where $M_{l^{\prime} l}^{\mathrm{D}}$ is a complex matrix. If the mass term (22) enter into Lagrangian, the total lepton number $L$ is conserved. Due to the conservation of $L$ in the case of the Dirac mass term there are no transitions into sterile states. For two neutrinos $\nu_{e}$ and $\nu_{\mu}$ the possible oscillations are $\nu_{e} \rightleftarrows \nu_{\mu}$ and $\bar{\nu}_{e} \rightleftarrows \bar{\nu}_{\mu}$, the same as in the Majorana case.

In 32] we have introduced the most general mass term. If we assume that $\nu_{l L}$ and $\nu_{l R}$ enter into the mass term and the total lepton number $L$ is not conserved for the mass term we obtain

$$
\begin{array}{r}
\mathcal{L}^{\mathrm{D}+\mathrm{M}}=-\frac{1}{2} \sum_{l, l^{\prime}}\left(\overline{\left.\nu_{l^{\prime} L}\right)^{c}} M_{l^{\prime} l}^{\mathrm{L}} \nu_{l L}-\sum_{l^{\prime} l^{\prime}} \bar{\nu}_{l^{\prime} R} M_{l^{\prime} l}^{\mathrm{D}} \nu_{l L}\right. \\
-\frac{1}{2} \sum_{l, l^{\prime}} \bar{\nu}_{l^{\prime} R} M_{l^{\prime} l}^{\mathrm{R}}\left(\nu_{l R}\right)^{c}+\text { h.c. }
\end{array}
$$

Here $M^{\mathrm{L}}$ and $M^{\mathrm{R}}$ are complex symmetrical matrices and $M^{\mathrm{D}}$ is a complex matrix. The Eq.(23) includes left-handed Majorana mass term, the Dirac mass term and the right-handed Majorana mass term. It is called the Dirac and Majorana mass term. 
For the mixing in the general case of $n$ flavors from (23) we have

$$
\begin{aligned}
\nu_{l L} & =\sum_{i=1}^{2 n} U_{l i} \nu_{i L} \\
\left(\nu_{l R}\right)^{c} & =\sum_{i=1}^{2 n} U_{\overline{l i}} \nu_{i L},
\end{aligned}
$$

where $U$ is an unitary $2 \mathrm{n} \times 2 \mathrm{n}$ mixing matrix, $\nu_{i}$ is the field of the Majorana neutrino with mass $m_{i}$. Now after the LEP experiments we know that $n=3$.

From (24) it follows that in the case of small $m_{i}$ transitions $\nu_{l} \rightarrow \nu_{l^{\prime}}$ (flavor-flavor) and $\nu_{l} \rightarrow \bar{\nu}_{l^{\prime} L}$ (flavor -sterile) are possible. Vacuum oscillations of the solar neutrinos in the general case of the Dirac and Majorana mass term were considered in 32. As it is well known the Dirac and Majorana mass term is the framework for the see-saw mechanism of neutrino mass generation.

In the seventies it was developed also the theory of neutrino oscillations in vacuum which is widely used today for analysis of the data of neutrino oscillation experiments.

In the case of neutrino mixing the lepton numbers $L_{e}, L_{\mu}$ and $L_{\tau}$ are not conserved. What are flavor neutrinos $\nu_{e}, \nu_{\mu}, \nu_{\tau}$ and corresponding antineutrinos in this case? From the very beginning we determine flavor neutrinos and antineutrinos as particles which take part in the standard CC weak processes with corresponding leptons. For example, neutrino which is produced together with $\mu^{+}$in the decay $\pi^{+} \rightarrow \mu^{+}+\nu_{\mu}$ is muon neutrino $\nu_{\mu}$, electron antineutrino $\bar{\nu}_{e}$ produces $e^{+}$in the process $\bar{\nu}_{e}+p \rightarrow e^{+}+n$ etc.

The states of flavor neutrinos are given by

$$
\left|\nu_{l}\right\rangle=\sum_{i} U_{l i}^{*}\left|\nu_{i}\right\rangle,
$$

where $\left|\nu_{i}\right\rangle$ is the state of neutrino with mass $m_{i}$, momentum $\vec{p}$ and energy $E_{i}=\sqrt{p^{2}+m_{i}^{2}} \simeq p+\frac{m_{i}^{2}}{2 p}$. Thus, flavor neutrinos are described by mixed coherent states.

The relation (25) is based on the assumption that neutrino mass-squared differences are so small that due to the uncertainty relation it is impossible to distinguish production (detection) of neutrinos with different masses. This condition can be presented in the form

$$
L_{o s c} \gg d
$$


where $\mathrm{d}$ is a quantum mechanical dimension of a neutrino source and

$$
L_{o s c}=4 \pi \frac{E}{\Delta m^{2}}
$$

is the oscillation length. If this condition is satisfied, neutrino cross sections and decay probabilities are given by the Standard Model.

If we apply now to the flavor states the evolution equation of the field theory

$$
i \frac{\partial|\Psi(t)\rangle}{\partial t}=\mathrm{H}|\Psi(t)\rangle
$$

we come to the standard expression for the transition probability

$$
\mathrm{P}\left(\nu_{l} \rightarrow \nu_{l^{\prime}}\right)=\left|\delta_{l l^{\prime}}+\sum_{i \geq 2} U_{l^{\prime} i} U_{l i}^{*}\left(e^{-i \Delta m_{i 1}^{2} \frac{L}{2 E}}-1\right)\right|^{2} .
$$

Here $L$ is the source-detector distance, $E$ is neutrino energy and $\Delta m_{i 1}^{2}=$ $m_{i}^{2}-m_{1}^{2}$.

Necessary condition for the observation of neutrino oscillations has the form

$$
\Delta m_{i 1}^{2} \frac{L}{E} \geq 1
$$

From this condition we see that experiments on the search for neutrino oscillations have enormous sensitivity to neutrino mass squared differences (for example, reactor experiments of the KamLAND type with $L \simeq 100 \mathrm{~km}$ and $E \simeq 1 \mathrm{MeV}$ are sensitive to $\Delta m^{2} \simeq 10^{-5} \mathrm{eV}^{2}$ etc)

For us this was the main reason and motivation for neutrino oscillations experiments: due to interference nature of the phenomenon of neutrino oscillations and possibility to perform neutrino experiments with large values of the parameter $\frac{L}{E}$ investigation of neutrino oscillations is extremely sensitive method to search for small $\Delta m^{2}$. This strategy brought success. We summarized it in the first review on neutrino oscillations published in 1977 33. Except papers of B.Pontecorvo, MNS and B.Pontecorvo and collaborators at that time it was published only a few papers on neutrino oscillations (34, 35, 36, 37. ).

At the end of the seventies a common interest to the problem of the neutrino mass and mixing started. It was connected with the appearance of the GUT models and the invention of the see-saw mechanism of the neutrino mass generation. Neutrino masses started to be considered as a signature 
of a new, beyond the Standard Model physics. At that time special reactor and accelerator neutrino experiments on the search for neutrino oscillations started. The investigation of matter effects in the case of neutrino mixing in the seventies [38] and disclosure of the MSW effect in the eighties 39] had very important impact on the field.

\section{Conclusion}

Today all existing neutrino oscillation data with the the exception of the data of LSND experiment [40], which need confirmation, are described by the three-neutrino mixing.

For neutrino oscillation parameters the following values were obtained [1, 3, 41]:

$$
\begin{gathered}
\Delta m_{21}^{2}=\left(8.2_{-0.5}^{+0.6}\right) \cdot 10^{-5} \mathrm{eV}^{2} ; \quad \tan ^{2} \theta_{12}=\left(0.40_{-0.07}^{+0.09}\right) \\
1.9 \cdot 10^{-3} \leq \Delta m_{32}^{2} \leq \\
3.0 \cdot 10^{-3} \mathrm{eV}^{2} ; \quad \sin ^{2} 2 \theta_{23} \geq 0.90 \\
\sin ^{2} \theta_{13} \leq 5 \cdot 10^{-2}
\end{gathered}
$$

Thus, neutrino oscillation parameters satisfy inequalities

$$
\Delta m_{21}^{2} \ll \Delta m_{32}^{2} ; \quad \sin ^{2} \theta_{13} \ll 1
$$

It follows from (32) (see 42) that the dominant transitions, governed by $\Delta m_{32}^{2}$, are $\nu_{\mu} \rightarrow \nu_{\tau}$ and $\bar{\nu}_{\mu} \rightarrow \bar{\nu}_{\tau}$. Dominant transitions, governed by $\Delta m_{21}^{2}$, are $\nu_{e} \rightarrow \nu_{\mu, \tau}$ and $\bar{\nu}_{e} \rightarrow \bar{\nu}_{\mu, \tau}$. This is a present-day picture of neutrino oscillations.

The main question to be answered what physics was discovered? What are implications of the discovered phenomenon? From my point of view to answer these fundamental questions we need to know

1. Are massive neutrinos Majorana or Dirac particles?

2. What is the neutrino mass spectrum (hierarchical, inverted, degenerate etc)?

3. What is the mass of the lightest neutrino ?

4. How many massive neutrinos exist in nature? Are sterile neutrinos exist? 
5. What is the value of the parameter $\sin ^{2} \theta_{13}$ ?

6. What is the value of CP phase?

7. What are precise values of oscillation parameters?

8. ...

I think that the first period of the investigation of neutrino properties is basically finished. In spite it took many years to discover neutrino oscillations, in a sense up to now we have been lucky. Seems that the next decisive step will be difficult and will require a lot of efforts. Existing data allows us to conclude that to probe the nature of neutrinos via the investigation of neutrinoless double $\beta$-decay is a challenge. It will be extremely difficult to observe this process if Majorana neutrino mass spectrum is hierarchical, which is a plausible possibility. The solution of other problems also look as a difficult and challenging task. After the discovery of neutrino masses and mixing we know, however, what to look for. This is a great advantage of the present stage.

The history of neutrino oscillations is an illustration of a complicated and thorny way of science.

- Correct pioneer ideas sometimes can have wrong basis or can be accompanied by wrong one.

- Courageous general ideas have good chances to be correct.

- Analogy is an important guiding principle in physics.

I will finish with the citation from the S.L. Glashow report [43] at the Venice "Neutrino Telescope Workshop" ( March 2003).

"...If only Bruno Pontecorvo could have seen how far we have come towards understanding the pattern of neutrino masses and mixing! Way back in 1963 he was among the first have envisaged the possibility of neutrino flavor oscillations. For that reason. the analog to the Cabibbo-Kobayashi-Maskawa matrix pertinent to neutrino oscillations should be known as the PMNS matrix to honor four neutrino visionaries: Pontecorvo, Maki, Nakagawa, and Sakata" 


\section{References}

[1] Super-Kamiokande Collaboration, S. Fukuda et al., Phys. Rev. Lett. 81, 1562 (1998); S. Fukuda et al., Phys. Rev. Lett. 82, 2644 (1999); S. Fukuda et al., Phys. Rev. Lett. 85, 3999-4003 (2000). E. Kearns Proceedings of 21th International Conference on Neutrino Physics and Astrophysics (Neutrino 2004), 13-19 June 2004, Paris, France.

[2] SNO collaboration, Q.R. Ahmad et al., Phys. Rev. Lett. 87, 071301 (2001) Phys.Rev.Lett. 89, 011301 (2002); nucl-ex/0204008. Phys.Rev.Lett 89, 011302 (2002); nucl-ex/0204009. nucl-ex/0309004.

[3] KamLAND Collaboration, T. Araki et al hep-ex/0406035, submitted to Phys.Rev.Lett.

[4] B. T. Cleveland et al., Astrophys. J. 496 (1998) 505.

[5] GALLEX Collaboration, W. Hampel et al., Phys. Lett. B 447 (1999) 127 ; GNO Collaboration, M. Altmann et al., Phys. Lett. B 490 (2000) 16 ; Nucl.Phys.Proc.Suppl. 91 (2001) 44.

[6] SAGE Collaboration, J. N. Abdurashitov et al., Phys. Rev. C 60 (1999) 055801 ; Nucl.Phys.Proc.Suppl. 110 (2002) 315;

[7] Super-Kamiokande Collaboration, S. Fukuda et al., Phys. Rev. Lett. 86 (2001) 5651;

[8] Soudan 2 Collaboration, W.W.M.Allison et al., Physics Letters B 449 (1999) 137;

[9] MACRO Collaboration, M.Ambrosio et al. hep-ex/0106049 Phys. Lett. B517 (2001) 59 M. Ambrosio et al. NATO Advanced Research Workshop on Cosmic Radiations, Oujda (Morocco), 21-23 March, 2001.

[10] K2K Collaboration, T. Nakaya, Proceedings of 21th International Conference on Neutrino Physics and Astrophysics (Neutrino 2004), 13-19 June 2004, Paris, France.

[11] B. Pontecorvo, J.Exptl. Theoret. Phys. 33 (1957) 549 [Sov. Phys. JETP 6 (1958) 429]. 
[12] B. Pontecorvo, J.Exptl. Theoret. Phys. 34 (1958) 247 [Sov. Phys. JETP 7 (1958) 172].

[13] E. Fermi, Ricerca Scientifica 2(1933) N12; Z.Physik 88 (1934) 161.

[14] F. Perrin, C.R. 197 (1933) 1625.

[15] G. Hanna and B. Pontecorvo, Phys. Rev. 75 (1949) 983; S. Curran et al Phil. Mag. 40 (1949) 53.

[16] L.D. Landau, Nucl.Phys.3 (1957) 127.

[17] T.D. Lee and C.N.Yang, Phys. Rev. 105 (1957) 1671.

[18] A. Salam, Nuovo Cimento 5 (1957) 299.

[19] C.S. Wu et al, Phys. Rev. 105 (1957) 1413.

[20] M. Goldhaber, L. Grodzins and A.W. Sunyar, Phys. Rev. 109 (1958) 1015 .

[21] R.P.Feynman and M.Gell-Mann, Phys. Rev.109 (1958) 193.

[22] E.C.G. Sudarshun and R. Marshak, Phys. Rev. 109 (1958) 1860.

[23] R. Davis, Bull. Am. Phys. Soc. ( Washington. meeting, 1959).

[24] F. Reines and C. Cowan, Phys.Rev. 113 (1959) 273.

[25] G. Danby, J.M. Gaillard, K. Goulianos, L.M. Lederman, M. Mistry, M. Schwartz and J. Steinberger, Phys. Rev.Lett. 9 (1962) 36.

[26] B. Pontecorvo, J. Exptl. Theoret. Phys. 53 (1967) 1717. [Sov. Phys. JETP 26 (1968) 984].

[27] Z. Maki, M. Nakagava and S. Sakata, Prog. Theor. Phys. 28 (1962) 870.

[28] G. Feinberg, Phys.Rev., 110 (1958) 1482.

[29] M. Gell-Mann and Levi, Nuovo Cimento, 10 (1960) 705.

[30] V. Gribov and B. Pontecorvo, Phys. Lett. B28 (1969) 493. 
[31] S.M. Bilenky and B. Pontecorvo, Phys. Lett. B61 (1976) 248; Yad. Fiz. 3 (1976) 603 .

[32] S.M. Bilenky and B. Pontecorvo, Lett. Nuovo Cim. 17 (1976) 569.

[33] S.M. Bilenky and B. Pontecorvo, Phys. Rep. 41 (1978) 225 .

[34] J. Bahcall and S. Frautschi, Phys. Lett. 29 (1969) 623.

[35] H. Fritzsch and P. Minkowski, Phys. Lett. B62 (1976) 72

[36] S. Eliezer and A. Swift, Nucl.Phys. B105 (1976) 45.

[37] S.M. Bilenky and B. Pontecorvo, JINR Preprint E2-10032, Dubna, 1976, Proceeding of Intern. Conf. on High Energy Physics, Tbilisi, July 1976.

[38] L. Wolfenstein, Phys. Rev. D 17 (1978)2369 ; Phys. Rev. D 20 (1979) 2634.

[39] S.P. Mikheyev and A.Yu. Smirnov, Yad. Fiz. 42 (1985) 1441 [Sov. J. Nucl. Phys. 42 (1985) 913 ]; Il Nuovo Cim. C 9 (1986) 17 ; Zh. Eksp. Teor. Fiz. 91 (1986) 7 [Sov. Phys. JETP 64(1986) 4].

[40] LSND Collaboration, A. Aguilar et al., Phys.Rev.D64 (2001) 112007; hep-ex/0104

[41] CHOOZ Collaboration, M. Apollonio et al., Phys. Lett. B 466415 (1999).

[42] S. M. Bilenky , C. Giunti and W. Grimus, Prog.Part.Nucl.Phys. 43 (1999) 1; hep-ph/9812360.

[43] S.L. Glashow Proceedings of the 10th International Workshop on Neutrino Telescopes, Venice, Italy, 11-14 Mar 2003, vol. 2, p. 611; hep-ph/0306100. 